\title{
Caracterização ao nascimento e nutricional dos prematuros em unidade intensiva de um hospital público
} Characterization at birth and nutritional of
premature newborns in the intensive unit of a
public hospital

\begin{abstract}
Elaine Priscila Pechepiura1, Márcia Helena de Souza Freire ${ }^{2}$, Karoline Petricio Martins $^{3}$, Magda Nanuck de Godoy Ribas Pinto ${ }^{4}$, Suellen da Rocha Lage Moraes ${ }^{5}$
\end{abstract}

1. ORCID: https://orcid.org/0000-0002-5431-0384. Enfermeira. Universidade Federal do Paraná, Curitiba, Paraná, Brasil. elainepechepiura7@gmail.com

2. 0000-0003-3941-3673. Enfermeira. Doutora em Saúde Pública. Professora adjunta. Universidade Federal do Paraná, Curitiba, Paraná, Brasil. marciahelenafreire@ gmail.com

3. ORCID: https://orcid.org/0000-0002-1251-5701. Enfermeira. Mestre em Enfermagem. Universidade Federal do Paraná, Curitiba, Paraná, Brasil. karolinepetricio@gmail.com

4. ORCID: https://orcid.org/0000-0001-8265-1584. Enfermeira. Mestre em Educação. Professora titular. Universidade Federal do Paraná, Curitiba, Paraná, Brasil. magda. ngh@gmail.com

5. ORCID: https://orcid.org/0000-0002-2468-9784. Enfermeira. Mestre em Enfermagem. Doutoranda em Enfermagem. Universidade Federal do Paraná, Curitiba, Paraná, Brasil. suellen_rl@hotmail.com

CONTATO: Elaine Priscila Pechepiura | Endereço: Avenida Ademar de Barros, 715 - Jardim Social, Campo Largo-PR, Brasil - Telefone: (41) 998018810. E-mail: elainepechepiura7@gmail.com

RESUMO A prematuridade está associada à mortalidade infantil, um problema de saúde pública global. $\mathrm{O}$ objetivo deste estudo foi caracterizar o perfil ao nascimento e nutricional de prematuros em unidade intensiva, de hospital público, Paraná. Estudo prospectivo, quantitativo, realizado de janeiro a abril de 2020. Total de 16 internações, prevalência do sexo masculino $(56,3 \%)$ e, nascimentos cirúrgicos $(62,5 \%)$. Metade dos recém-nascidos eram prematuros extremos, muito prematuros e, extremo baixo peso; todos, com diagnóstico 
CID-10 de desconforto respiratório do recém-nascido; Apgar $\geq 7$ no $5^{\circ}$ minuto (75,0\%); ocorreu um óbito. Nutrição parenteral e/ou enteral até 72 horas de vida ocorrida para 13 recém-nascidos prematuros $(81,3 \%) ; 14$ prematuros extremos com nutrição parenteral até o terceiro dia $(87,5 \%)$; e, todos os muito prematuros $(n=8)$ com nutrição enteral. $\mathrm{O}(\mathrm{re})$ conhecimento do cenário propicia a qualificação da atenção às gestantes e aos recém-nascidos, quanto ao planejamento do cuidado seguro e, ao estado nutricional, repercutindo na sobrevida.

DESCRITORES: Recém-nascido Prematuro. Unidades de Terapia Intensiva Neonatal. Assistência Alimentar. Enfermagem Perinatal.

\begin{abstract}
Prematurity is associated to child mortality, a public health problem. The aim of this study was to characterize the birth and nutritional profile of preterm infants in an intensive care unit at a public hospital. Prospective, quantitative study, carried out from January to April 2020. Total of 16 hospitalizations with male prevalence $(56.3 \%)$ and surgical births $(62.5 \%)$. Half of the newborns were extremely premature, very premature, and extremely low birth weight. All of newborns with a diagnosis of respiratory distress of the newborn and Apgar score $\geq 7$ in the 5th minute $(75.0 \%)$. During the data collection, there was a death. Parenteral and/or enteral nutrition up to 72 hours of life occurred for 13 premature newborns $(81.3 \%) 14$ extremely preterm infants with parenteral nutrition until the third day of life $(87.5 \%)$ and all very premature $(n=8)$ with enteral nutrition. The recognition of the scenario provides the qualification of care to pregnant women and newborns, regarding the planning of safe care and nutritional status affecting survival.
\end{abstract}

DESCRIPTORS: Infant, Premature. Intensive Care Units, Neonatal. Food Assistance. Perinatal Nursing.

\title{
INTRODUÇÃO
}

prematuridade destaca-se como um problema de saúde pública pois, suas
complicações, de diversas ordens, constituem-se como a principal causa de morte
neonatal e, a segunda entre as crianças menores de cinco anos ${ }^{1}$ O nascimento prematuro
é o ocorrido antes de 37 semanas completas de gestação e, é dividido em: prematuro
extremo, menos de 28 semanas; muito prematuro, 28 a menos de 32 ; e, prematuro
moderado ou tardio, 32 a menos de $37^{2}$.
No Brasil, a mortalidade neonatal precoce ( 0 a 6 dias de vida completos) responde por
$50 \%$ das mortes infantis, e reflete a qualidade da atenção prestada no pré-natal, durante 
e após o parto. Dado que reforça a importância das políticas públicas de atenção à mulher e ao recém-nascido $(\mathrm{RN})^{3}$. O período neonatal compreende desde o nascimento até o $27^{\circ}$ dia de vida completo, sendo considerada uma fase de adaptação e vulnerabilidade ${ }^{4}$.

Quanto aos nascimentos prematuros, a cada ano, em todo o mundo, são estimados na ordem de 15 milhões. No Brasil, cerca de $12 \%$ dos nascimentos ocorrem antes de 37 semanas de gestação ${ }^{5}$. Embora sua etiologia ainda não seja totalmente determinada, as causas mais comuns de nascimento prematuro incluem gravidez gemelar, antecedente de parto prematuro, pré-eclâmpsia e controle pré-natal insuficiente ou ausente ${ }^{6}$.

Contudo, sabe-se que o aprimoramento do conhecimento científico a respeito da saúde e do melhor desenvolvimento do RN, bem como, a inclusão de tecnologias de cuidado às atividades hospitalares, podem modificar o prognóstico e, a sobrevida do RN de alto risco internado em Unidade de Terapia Intensiva Neonatal (UTIN)7.

Na ocorrência do nascimento prematuro, a nutrição até então fornecida pela placenta é interrompida. Então, o crescimento adequado, próximo das taxas intrauterinas à cada idade gestacional, passa a depender da introdução precoce de nutrientes, para a manutenção da massa corporal e da densidade óssea; prevenção de septicemia, enterocolite necrosante (ECN) e displasia broncopulmonar; e, otimização do desenvolvimento neurológico ${ }^{8}$.

Este estudo com recém-nascidos prematuros foi desenvolvido considerando a necessidade, devida à sua expressão entre as causas de mortes infantis e interesse acadêmico, em conhecer aspectos desta população de prematuros, identificando variáveis de nascimento, gestacionais, de diagnóstico médico, de assistência e nutricionais, como o início e o tipo do suporte nutricional ao RN pré-termo (RNPT) internado em UTIN. O mesmo não objetivou ser um estudo delineado com método mais denso e complexo para gerar uma evidência de elevado nível, mas, (re)conhecer o cenário, gerando uma evidência loco-regional.

Assim, com o intuito de gerar subsídios que possam contribuir com a gestão dos leitos e o planejamento do cuidado e da unidade investigada, bem como, com a melhoria da sobrevida dessa população, realizou-se o presente estudo. O objetivo geral foi: caracterizar o perfil de nascimento e nutricional dos recém-nascidos prematuros internados em Unidade de Terapia Intensiva de um hospital público de ensino, de Curitiba, estado do Paraná, Brasil.

\section{MÉTODO}

Trata-se de estudo prospectivo do tipo descritivo, de abordagem quantitativa, estruturado segundo as recomendações do checklist Strengthening the Reporting of Observational Studies in Epidemiology (STROBE) ${ }^{9}$. O cenário foi a UTIN de um grande 
hospital público, de ensino, localizado na cidade de Curitiba, estado do Paraná, inaugurado em 1961. Atualmente é o maior prestador de serviços do Sistema Único de Saúde (SUS) do estado e, referência ambulatorial, para internação, parto e puerpério, para gestantes e RN de alto risco por possuir estrutura tecnológica, instrumental e recursos humanos para atendimento de alta complexidade ${ }^{10}$.

Embora o quantitativo dos leitos abertos oscile devido à necessidades internas, na época da coleta de dados (dezembro/2019 a abril/2020) a Unidade Neonatal apresentavase com 30 leitos, sendo 10 leitos de UTIN e 20 de Unidade de Cuidados Intermediários Convencionais (UCINCo). A Unidade Canguru (UCINCa) encontrava-se fechada devido à pandemia de COVID-19. Neste período, as taxas de ocupação da UTIN e da UCIN mantiveram-se permanentemente próximas a $100 \%$.

Este estudo é um recorte de um projeto de pesquisa maior intitulado "Protocolo de Manuseio Mínimo para recém-nascidos prematuros em Unidade de Terapia Intensiva Neonatal de Hospital Público". Foram utilizados dados secundários, procedentes de prontuários hospitalares, das internações de RNPT, <32 semanas de IG, durante os meses de coleta. Na intenção de obter resultados que pudessem ser cotejados com o estado de saúde dos RNPT, também foram coletados dados maternos que constavam dos prontuários dos RNPT, que são também apresentados. Cada RNPT participante foi incluído, após o convite e esclarecimento do objetivo do estudo à sua mãe/pai ou responsável e, assinatura do Termo de Consentimento Livre e Esclarecido (TCLE) ou de Assentimento, pelo mesmo. Mesmo que a abordagem ao RN não tenha sido direta ou implicado em qualquer manipulação. Foram apenas extraídos dados de interesse do prontuário do mesmo.

As variáveis dos RNPT incluídas, segundo a sua definição, características ou categorias, foram: via de nascimento (cirúrgica e vaginal); sexo (feminino e masculino); Apgar (maior ou igual a 7, entre 6 e 4 e entre 3 e 0 ); idade gestacional - prematuro extremo (menor de 28 semanas) e muito prematuro (entre 28 a menos de 32 semanas); peso ao nascimento - extremo baixo peso (menos de $1000 \mathrm{~g}$ ), muito baixo peso (menos de1500 g) e baixo peso (menos de $2500 \mathrm{~g}$ ); reanimação em sala de parto - pressão positiva contínua de vias aéreas (CPAP), ventilação com pressão positiva (VPP) e intubação orotraqueal (IOT); e diagnósticos médicos (extraídos segundo a Classificação Estatística Internacional de Doenças e Problemas Relacionados à Saúde $-10^{\mathrm{a}}$ Revisão, CID-10) $)^{11}$.

As variáveis maternas foram: idade cronológica (registradas nos prontuários dos RNPT); escolaridade (sem instrução e fundamental incompleto, fundamental completo e médio incompleto, médio completo e superior incompleto, superior completo, e não informado); raça/cor (branca, preta, amarela, parda e indígena); tipo de gravidez (única 
e múltipla); intercorrências gestacionais (registradas nos prontuários dos RNPT); e, medicações (utilizadas durante a gestação, trabalho de parto e parto).

Ressalta-se que as variáveis diagnósticos médicos, intercorrências gestacionais e medicações, admitiram mais de uma resposta ou categoria.

Adotaram-se como critérios para a inclusão dos prontuários dos RN que os mesmos tivessem nascido na instituição em estudo; estivessem com idade gestacional <32 semanas; e, que permanecessem na instituição durante as primeiras 72 horas de vida; não apresentando patologias associadas. Foram excluídos os prontuários dos RN que apresentaram anomalia congênita incompatível com a vida e, anomalia congênita de trato gastrointestinal, devido intervir na análise do suporte nutricional.

A organização dos dados foi realizada com o apoio do Microsoft Office Excel $囚$, realizouse análise descritiva de frequência numérica, proporção, média e mediana.

A pesquisa que deu origem a este estudo foi aprovada pelo Comitê de Ética em Pesquisa em Seres Humanos do Hospital de Clínicas da Universidade Federal do Paraná, sob CAAE 25514119.7.0000.0096. Foram respeitadas as recomendações da Resolução n 466 de 2012 do Conselho Nacional de Saúde, que aprova as diretrizes e normas regulamentadoras de pesquisas envolvendo seres humanos ${ }^{12}$.

\section{RESULTADOS}

A amostra constituiu-se por 16 prontuários de RNPT, sendo que nove deles $(56,3 \%)$ do sexo masculino; dez (62,5\%) nascidos de parto cesariana; oito $(50,0 \%)$ prematuros extremos; oito $(50,0 \%)$ muito prematuros; oito $(50,0 \%)$ com peso ao nascer extremamente baixo; e, dez $(62,5 \%)$ utilizaram CPAP em sala de parto. Os dados encontram-se na Tabela 1.

A idade gestacional ao nascimento variou entre 25 e 31 semanas e seis dias, com média de 28 semanas e dois dias e mediana de 28 semanas. O peso ao nascimento variou de $530 \mathrm{~g}$ a $1735 \mathrm{~g}$, com média de $1075 \mathrm{~g}$ e mediana de $1055 \mathrm{~g}$. O escore de Apgar teve como menores pontuações zero e três, no $1^{\circ}$ e $5^{\circ}$ minuto, respectivamente. Já a média do Apagar foi de 4,8 ( $1^{\circ}$ minuto $)$ e $7,6\left(5^{\circ}\right.$ minuto $)$ e a mediana foi de $5,5\left(1^{\circ}\right.$ minuto) e 8,5 ( $5^{\circ}$ minuto). Ocorreu um óbito $(6,3 \%)$.

Quanto à prontidão do encaminhamento para atendimento na UTIN, foi observado que o tempo médio entre o nascimento e a admissão na UTIN foi de 30 minutos e 45 segundos, com mediana de 27 minutos.

Quanto as variáveis maternas, ressalta-se que como houve uma mãe com nascimento de dois gêmeos, o número total de mulheres mães apresentado é 15, destas $11(73,3 \%)$ possuíam entre 20 e 34 anos e, quatro (26,7\%) eram $\leq 19$ anos ou $\geq 35$ anos; variando 
assim a idade materna entre 17 a 36 anos, com média de 28,1 e mediana de 28 anos.

Quanto à raça/cor, oito $(53,3 \%)$ mães eram brancas e, $6(40,0 \%)$ pardas. Quanto ao grau de instrução sete $(46,7 \%)$ concluíram o ensino médio, cinco $(33,3 \%)$ o fundamental e, uma $(6,7 \%)$, o superior.

Tabela 1. Distribuição ( $n$ e \%) dos recém-nascidos pré-termo $(n=16)$ e das mães $(n=15)$ segundo variáveis selecionadas para o estudo, Unidade de Terapia Intensiva Neonatal, hospital público de ensino, Curitiba - PR, 2020

\begin{tabular}{|c|c|c|}
\hline Variáveis neonatais & (n) & $(\%)$ \\
\hline \multicolumn{3}{|l|}{ Via de nascimento } \\
\hline Cesariana & 10 & 62,5 \\
\hline Vaginal & 6 & 37,5 \\
\hline \multicolumn{3}{|l|}{ Sexo } \\
\hline Feminino & 7 & 43,8 \\
\hline Masculino & 9 & 56,3 \\
\hline \multicolumn{3}{|l|}{ Apgar $1^{\circ}$ minuto } \\
\hline$\geq 7$ & 6 & 37,5 \\
\hline 6 a 4 & 5 & 31,3 \\
\hline 3 a 0 & 5 & 31,3 \\
\hline \multicolumn{3}{|l|}{ Apgar $5^{\circ}$ minuto } \\
\hline$\geq 7$ & 12 & 75,0 \\
\hline 6 a 4 & 3 & 18,8 \\
\hline 3 a 0 & 1 & 6,3 \\
\hline \multicolumn{3}{|l|}{ Idade gestacional (semanas) } \\
\hline Prematuro extremo $(<28)$ & 8 & 50,0 \\
\hline Muito prematuro $(28 \mathrm{a}<32)$ & 8 & 50,0 \\
\hline \multicolumn{3}{|l|}{ Peso ao nascimento (gramas) } \\
\hline Extremo Baixo Peso $(<1000)$ & 8 & 50,0 \\
\hline Muito Baixo Peso $(<1500)$ & 6 & 37,5 \\
\hline Baixo Peso $(<2500)$ & 2 & 12,5 \\
\hline \multicolumn{3}{|l|}{ Reanimação em sala de parto } \\
\hline Pressão positiva contínua de vias aéreas (CPAP) & 10 & 62,5 \\
\hline Ventilação com pressão positiva (VPP) & 7 & 43,8 \\
\hline Intubação orotraqueal (IOT) & 5 & 31,3 \\
\hline \multicolumn{3}{|l|}{ Variáveis maternas $(n=15)^{*}$} \\
\hline \multicolumn{3}{|l|}{ Idade (anos) } \\
\hline$\leq 19$ & 1 & 6,7 \\
\hline 20 a 34 & 11 & 73,3 \\
\hline$\geq 35$ & 3 & 20,0 \\
\hline \multicolumn{3}{|l|}{ Raça/cor } \\
\hline Branca & 8 & 53,3 \\
\hline Parda & 6 & 40,0 \\
\hline Não informada & 1 & 6,7 \\
\hline
\end{tabular}




\section{Escolaridade}

Sem instrução e fundamental incompleto $\quad 1 \quad 6,7$

Fundamental completo e médio incompleto $\quad 5 \quad 33,3$

$\begin{array}{lll}\text { Médio completo e superior incompleto } & 7 & 46,7\end{array}$

$\begin{array}{lll}\text { Superior completo } & 1 & 6,7\end{array}$

Não informado 116,7

*Houve a ocorrência de uma gestação múltipla, dois RN gêmeos.

Fonte: Elaborada pelas autoras (2020).

Quase a metade das mulheres $(46,7 \%)$ realizaram 6 ou mais consultas de pré-natal, com média de 6,7 e mediana 6 . No período, ocorreu apenas uma $(6,7 \%)$ gestação múltipla. A hipertensão materna foi a intercorrência gestacional mais representativa $(42,9 \%$; $n=6)$, seguida pelo descolamento prematuro de placenta $(21,4 \% ; n=3)$, infecção do trato urinário $(14,3 \% ; n=2)$, diabetes mellitus $(14,3 \% ; n=2)$ e ruptura prematura de membranas $(14,3 \%$; n=2). $O$ uso de corticoides antenatal foi registrado em $78,6 \%$ dos prontuários.

$\mathrm{Na}$ Tabela 2, relacionam-se os 53 diagnósticos médicos obtidos dos prontuários dos RNPT, com visibilidade para cinco Capítulos e, 17 Categorias da CID-10, sendo que um RNPT pode ter recebido mais de um deles. O Capítulo mais representativo foi o XVI Algumas afecções originadas no período perinatal. As categorias mais recorrentes foram a P22 - Desconforto respiratório do recém-nascido (100,0\%; n=16) e a P59 - Icterícia neonatal devida a outras causas e às não especificadas $(50,0 \% ; n=8)$.

Tabela 2. Diagnósticos dos recém-nascidos pré-termo segundo Capítulos e Categorias da Classificação Internacional de Doenças, $10^{\mathrm{a}}$ Revisão, Unidade de Terapia Intensiva Neonatal, hospital público de ensino, Curitiba - PR, 2020

\section{Capítulos e Categorias da CID-10*}

IV - Doenças endócrinas, nutricionais e metabólicas

E87 - Outros transtornos do equilíbrio hidroeletrolítico e ácido-básico IX - Doenças do aparelho circulatório

127 - Outras formas de doença cardíaca pulmonar $\mathrm{XVI}$ - Algumas afecções originadas no período perinatal

P05 - Crescimento fetal retardado e desnutrição fetal

P21 - Asfixia ao nascer

P22 - Desconforto respiratório do recém-nascido

P26 - Hemorragia pulmonar originada no período perinatal

P28 - Outras afecções respiratórias originadas no período perinatal

P36 - Septicemia bacteriana do recém-nascido

P59 - Icterícia neonatal devida a outras causas e às não especificadas

P61 - Outros transtornos hematológicos perinatais

$\mathrm{XVI}$ - Algumas afecções originadas no período perinatal (n) $(\%)^{\star *}$

212,5

16,3

$4 \quad 25,0$

212,5

16100,0

16,3

212,5

212,5

850,0

16,3 
P70 - Transtornos transitórios do metabolismo dos carboidratos específicos do feto e do recém-nascido

P71 - Transtornos transitórios do metabolismo do cálcio e do magnésio do período neonatal

P77 - Enterocolite necrotizante do feto e do recém-nascido

P83 - Outras afecções comprometendo o tegumento específicas do

feto e do recém-nascido

XVIII - Sintomas, sinais e achados anormais de exames clínicos e de laboratório, não classificados em outra parte

R34 - Anúria e oligúria

R94 - Resultados anormais de estudos de função

16,3

$4 \quad 25,0$

XXI - Fatores que influenciam o estado de saúde e o contato com os serviços de saúde

Z20 - Contato com e exposição a doenças transmissíveis

TOTAL $3 \quad 18,8$

*Um RNPT pode ter recebido mais de um diagnóstico médico, todos foram relacionados.

**Proporção atribuída sob o total de crianças que recebeu o diagnóstico.

Fonte: Elaborada pelas autoras (2020).

Quanto à nutrição dos RNPT nas primeiras 24 horas de vida, todos receberam, por via endovenosa, soluções prontas com glicose, aminoácidos e cálcio que, na instituição em estudo, recebem o nome de Plano Básico. Com 72 horas, a oferta desta solução já havia decrescido para $25,0 \%$ dos RNPT, concomitante ao acréscimo de nutrição parenteral (NP) e/ou nutrição enteral (NE) (Gráfico 1).

Gráfico 1. Nutrição dos recém-nascidos pré-termo segundo tipo e tempo (horas) de vida, Unidade de Terapia Intensiva Neonatal, hospital público de ensino, Curitiba - PR, 2020

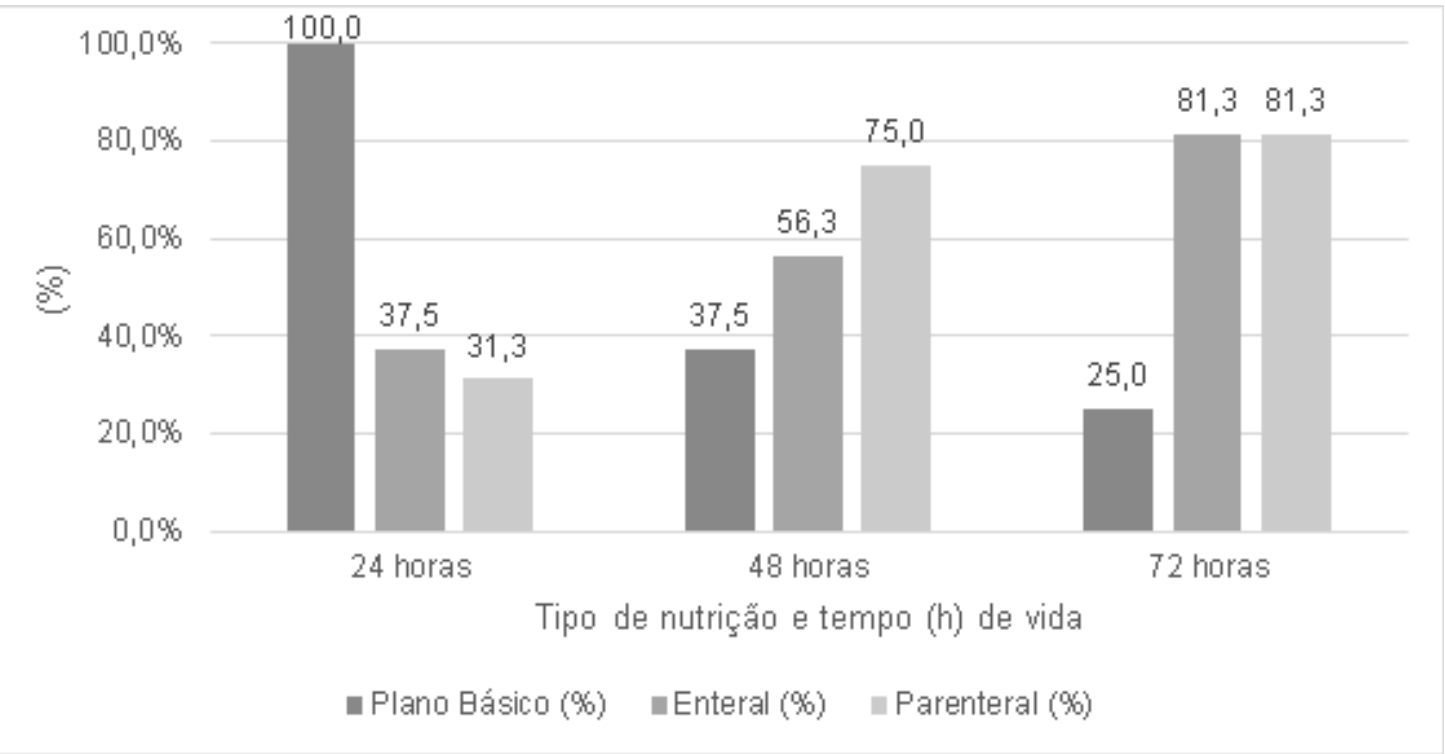

Fonte: Elaborado pelas autoras (2020). 
Com foco exclusivo aos RNPT extremos, $87,5 \%(n=14)$ deles, com 48 horas de vida, recebiam NP, como mostra o Gráfico 2. Com esta mesma idade, $100 \%(n=16)$ dos $\mathrm{RN}$ muito prematuros, recebiam NE, associada ou não à NP (Gráfico 3).

Gráfico 2. Nutrição dos recém-nascidos prematuros extremos (<28 semanas de idade gestacional) segundo a via de oferta, Unidade de Terapia Intensiva Neonatal, hospital público de ensino, Curitiba - PR, 2020

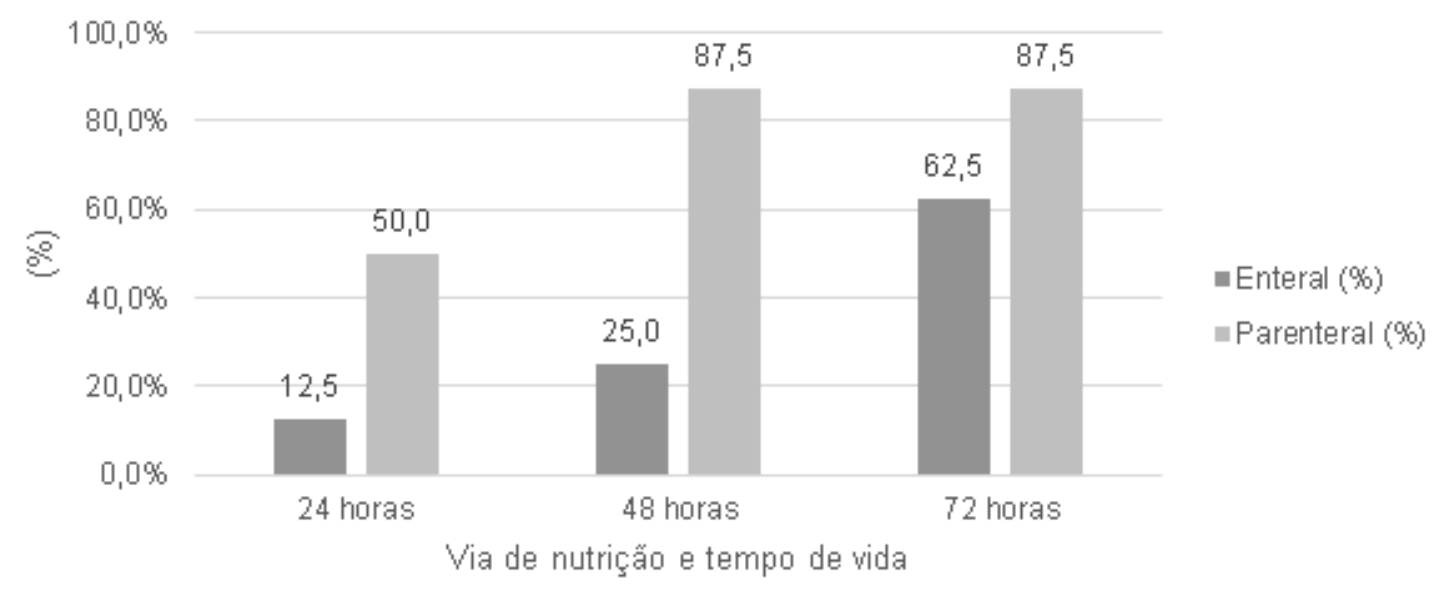

Fonte: Elaborado pelos autores (2020).

Gráfico 3. Nutrição dos recém-nascidos muito prematuros (de 28 a $<32$ semanas de idade gestacional) segundo a via de oferta, Unidade de Terapia Intensiva Neonatal, hospital público de ensino, Curitiba - PR, 2020



Fonte: Elaborado pelas autoras (2020).

\section{DISCUSSÃO}

Os RNPT, mundialmente, representam uma proporção de $11,1 \%$ dos nascimentos, e a prematuridade é responsável, direta ou indiretamente, por elevados coeficientes de morbidade e mortalidade infantil (MI). O Brasil está entre os dez países com as maiores taxas de nascimento prematuro. O cuidado adequado em UTIN visa reduzir os índices de morbimortalidade contudo, a baixa cobertura de cuidados intensivos neonatais de alta qualidade tem sido o maior motivo de óbito neonatal ${ }^{13-14}$. Nesse sentido, a prontidão no 
atendimento é imprescindível, quanto mais reduzido o tempo entre o encaminhamento - admissão - e início dos cuidados, melhores serão as chances de sobrevida. Esse tempo deve ser monitorado e, sempre que necessário, otimizado, nos serviços de UTIN, em prol das chances de prevenção de quaisquer danos ao RNPT. No presente estudo o tempo mediano foi inferior a 30 minutos, consideram-se que o estudo incluiu apenas nascidos na própria instituição.

Sabe-se que as intervenções de rotina, incluindo o tipo de parto, associam-se aos maiores gastos, sobretudo públicos, à mortalidade e, morbidades evitáveis. $\mathrm{O}$ aumento contínuo de cesarianas no Brasil, que hoje tem uma das maiores taxas do mundo, superou em muito a proporção de partos via vaginal no país. O estudo evidencia um número quatro vezes maior (62,5\%) do que o recomendado pela Organização Mundial da Saúde (OMS), que preconiza como taxa ideal de nascimentos cirúrgicos, entre 10 e $15 \%{ }^{15}$. Sabe-se que os partos cirúrgicos quando indicados por motivos médicos justificados, reduzem efetivamente a morbimortalidade materna e perinatal, em contrapartida, quando não necessários, acarretam riscos adicionais imediatos e, a longo prazo ${ }^{15-16}$. Destaca-se, contudo, que o parto cirúrgico neste estudo pode ter ocorrido pela necessidade e/ou urgência, não foram analisadas as justificativas e motivos nos prontuários maternos.

A possibilidade de agendar a data do parto e contar com um profissional já conhecido, aliada à percepção de maior segurança, são fatores que, em parte, explicam a elevação do número de cesarianas eletivas ${ }^{17}$. A cesariana iatrogênica tem sido associada ao nascimento pré-termo, ao aumento da morbidade respiratória neonatal, às internações em UTIN e, à necessidade de ventilação mecânica. Bem como, pode ser responsável por casos de morbidade neonatal near miss, caracterizado como "evento mórbido que quase resultou na morte do recém-nascido nos primeiros 28 dias de vida", visto que esses RN apresentam maior risco quando comparados com os nascidos por parto vaginal ${ }^{18}$.

Dados concordantes são os gerados em um estudo realizado em Porto Alegre, foi identificado que $56,2 \%$ dos RNPT nasceram de parto cirúrgico ${ }^{17}$. Estudo realizado no Recife, também identificou a cesariana como principal via de parto $(76,4 \%)$ entre os casos de near miss materno ${ }^{20}$. O near miss materno é definido como "mulheres que no ciclo gravídico puerperal estiveram muito próximo de morrer e sobreviveram"20.

A pesquisa Nascer no Brasil, realizada com 23.940 puérperas, demonstrou que a preferência das gestantes pelo parto cirúrgico aumentou ao longo da gestação, 27,6\% das entrevistadas referiam preferência pela cesárea no início da gestação e, ao seu final, um terço já havia decidido pela sua realização e ainda que, $51,5 \%$ delas a apresentaram como via de parto final ${ }^{17}$.

A transição da vida fetal para a extrauterina envolve múltiplos mecanismos de adaptação biológica, com participação de vários sistemas e órgãos. Um exemplo é o 
estabelecimento da respiração para a oxigenação do RN. As altas taxas de cesarianas e a influência nos mecanismos fisiológicos naturais implicam em riscos, evidenciado através das altas taxas de prematuridade, associadas a distúrbios respiratórios, à necessidade de assistência para iniciar a respiração bem como, de intubação e reanimação ${ }^{14,21}$.

Distúrbios respiratórios, decorrentes da má formação do sistema respiratório, foram significativamente observados neste estudo. Dado corroborado com um estudo realizado na UTIN de uma maternidade de hospital público, no interior de Minas Gerais, entre 2016 e 2018. Na qual, foi demonstrado que a síndrome respiratória foi a principal causa de complicação para internação dos neonatos $(36,4 \%)$, os quais, em sua maioria, nascidos de parto cirúrgico e pré-termos ${ }^{14}$.

Em relação ao sexo, convergente com os resultados apresentados, o Instituto Brasileiro de Geografia e Estatística (IBGE) apontou predomínio de nascidos vivos do sexo masculino no ano de $2018(51,2 \%)^{22}$. Como consequência observa-se ocorrência de maior número de internações de RNPT do sexo masculino. Cenário semelhante ao da pesquisa Nascer no Brasil, que apresentou $51,8 \%$ nascimentos masculinos ${ }^{23}$. Outro estudo também evidenciou leve predomínio do sexo masculino $(53,2 \%)$ de entre os nascimentos ${ }^{13}$. Nestes estudos, entretanto, se chama atenção para o fato de que foram incluídos RNPT e RN a termo.

Quanto à reanimação em sala de parto, identificou-se que todos os RNPT tiveram necessidade de algum tipo de manobra, a maioria necessitou de CPAP, contudo também receberam VPP e, uma menor proporção, culminou com necessidade de intubação traqueal. A aplicação de CPAP ajuda a manter os alvéolos não colapsados, evitando atelectrauma, é indicada em RNPT <34 semanas de IG que apresentem respiração espontânea, frequência cardíaca $>100$ batimentos por minuto $(\mathrm{bpm}) \mathrm{e}$, desconforto respiratório ou Saturação de Oxigênio (SatO ${ }^{2}$ ) baixa. Quando comparado o uso de CPAP com a intubação traqueal, verifica-se redução da necessidade de ventilação mecânica e de surfactante exógeno, bem como, da dependência de oxigênio e, do óbito hospitalar ${ }^{24}$.

Já a VPP é indicada na ocorrência de apneia e/ou respiração irregular e/ou bradicardia e, deve ser iniciada nos primeiros 60 segundos de vida. Inicialmente ventila-se com máscara facial e, quando não efetiva, indica-se a intubação ${ }^{24}$.

O óbito ocorrido no estudo foi de um RN com 25 semanas, e incidiu no período neonatal precoce. Atualmente, principal componente da MI. Entre as principais causas de óbito, a prematuridade responde por cerca de $1 / 3$ dos casos, e os óbitos se concentram entre os prematuros (extremos) e com (extremo) baixo peso ${ }^{23}$.

Quanto à IG e ao peso de nascimento, a proporção de prematuros extremos e muito prematuros foi a mesma $(50,0 \%)$, coincidente à de peso extremamente baixo. Outros 
estudos também demonstraram relação direta entre a IG e o peso ao nascer, quanto menor o tempo de desenvolvimento intraútero, menor será o peso do RN, também resultante de outras condições, como o crescimento intrauterino restrito, com influência na morbimortalidade neonatal ${ }^{13,19}$.

Outra variável que impacta no prognóstico e nas condutas com o RNPT, são os valores do índice de Apgar. O presente estudo teve $25,0 \%$ dos RNPT com valor inferior a sete no $5^{\circ}$ minuto. Essa pontuação é essencial para identificação da necessidade de algum tipo de intervenção nos primeiros minutos de vida, sendo que valor inferior a sete indica prognóstico ruim ${ }^{14}$.

Dois estudos realizados no município de Porto Alegre, um estudo de coorte com 419 $\mathrm{RN}$ de muito baixo peso e, um do tipo caso-controle, identificaram os fatores perinatais associados à mortalidade neonatal precoce e na sala de parto, e os fatores maternos e neonatais associados à prematuridade. Entre os fatores apontados relacionam-se: as menores IG; os menores pesos ao nascimento; e, os baixos índices de Apgar ${ }^{3,13}$.

O nascimento prematuro é considerado também como critério para uma urgência nutricional, visto que a oferta de nutrientes é interrompida e, seu fornecimento tardio ou insuficiente leva à desnutrição e, consequente, déficit de crescimento, além de outras consequências a curto e longo prazo. Assim, visando a promoção do adequado crescimento, recomenda-se iniciar o suporte nutricional logo após o nascimento ${ }^{25}$.

Estudos têm demonstrado que o início precoce de aminoácidos parenterais é bem tolerado, assim como à introdução de lipídeos com 24 horas de vida, devido à instabilidade clínica e à imaturidade gastrointestinal do RNPT, as quais dificultam a oferta por via enteral. A associação da NE mínima, preferencialmente nas primeiras 72 horas, estimula o funcionamento intestinal, melhora o crescimento e, reduz a incidência de sepse, a osteopenia e o tempo de internação, sem aumentar o risco de ECN ${ }^{25}$.

A microbiota intestinal tem papel fundamental no desenvolvimento das funções gastrointestinais pós-natais e, consequentemente, na redução do risco de infecção. Sua variabilidade é dependente de condições como tipo de parto, grau de imaturidade, uso de antibióticos, oferta de leite materno ou fórmula láctea, entre outras ${ }^{26}$.

O leite materno tem papel importante no estabelecimento da microbiota intestinal, associa-se à maior diversidade microbiana, favorecendo a prevenção de sepse, ECN e outras doenças. A longo prazo, previne obesidade, Diabetes Tipo 2, inflamação intestinal crônica, distúrbios autoimunes, alergia, Síndrome do Intestino Irritável e, gastroenterite alérgica. Em contrapartida, o uso de antibióticos e a demora no início da alimentação enteral promovem a redução da diversidade e da quantidade de microrganismos que colonizam o trato gastrointestinal, aumentando o risco de doenças ${ }^{26}$. 
Com relação ao tempo de início da nutrição, em estudo realizado nos Estados Unidos, ao se avaliar o início precoce da NP, observou-se melhora do ganho ponderal na alta ${ }^{27}$. Estudo alemão também demonstrou que a NE precoce está associada ao melhor crescimento ${ }^{28}$. Os dados encontrados no presente estudo são concordantes com as evidências teóricas sobre a importância da nutrição nessa população.

No que concerne às variáveis sociodemográficas maternas, dividiram-se entre pequena prevalência de brancas, e pardas. A idade teve média e mediana quase concordantes, próximas a 28 anos, mulheres adultas jovens, em idade reprodutiva, com no mínimo oito anos de estudo. E, às variáveis obstétricas, a maioria passou por um número próximo ao ideal de consultas pré-natal, com média e mediana próximas de seis. O início precoce (até a $16^{a}$ semana gestacional) ou tardio não pôde ser avaliado por falta dessa informação nos prontuários dos RNPT. Salienta-se a relevância das informações maternas para análise das condições dos RNPT.

Aponta-se que o IBGE, no censo de 2010, para a cidade de Curitiba, apresentou 76,3\% de mulheres brancas ${ }^{29}$. Outro estudo também identificou predomínio da raça branca $(75,7 \%)$ dentre as mães de RNPT tardios ${ }^{19}$. Em contrapartida, na pesquisa Nascer no Brasil, realizada com entrevistas às puérperas, $64,7 \%$ das mulheres se autodeclararam pardas ou pretas ${ }^{17}$. Outro estudo, realizado na cidade de São Paulo, também apresentou como raça predominante a parda ${ }^{30}$. É relevante destacar que a variável raça/cor de um RN deve ser preenchida na Declaração de Nascimento mediante a declaração da mulher e, não com avaliação de profissional de saúde.

$\mathrm{Na}$ pesquisa Nascer no Brasil, dados semelhantes foram encontrados no que se refere à idade materna, escolaridade e pré-natal. A maior parte das mães $(70,8 \%)$ apresentavam-se entre 20 e 34 anos de idade (variação entre 12 a 46 anos) e, a média de idade foi de 25,6 anos. Apresentavam ensino superior $8,8 \%$ delas ${ }^{17,23}$. Realizaram seis ou mais consultas de pré-natal $73,1 \%$ das mulheres ${ }^{31}$.

Outro estudo caracterizou os fatores sociodemográficos, obstétricos e as intercorrências obstétricas das mães de prematuros tardios. Como resultado houve prevalência de mães fora das faixas etárias extremas. A média de idade foi cerca de 27 anos, com idades mínimas e máximas de 14 e 44 anos, a maioria (quase 55\%) com mais de oito anos de ensino, e cerca de $59 \%$ realizaram seis ou mais consultas de pré-natal ${ }^{19}$. Sobre as características das gestantes com parto prematuro, um estudo apontou que as mulheres apresentavam entre 14 e 31 anos, com média de 24,9 $\pm 6,9$ anos, 34,4\% estavam na faixa etária considerada de risco pelo MS (<20 ou >35 anos) e, 41,8\% finalizaram o ensino médio ${ }^{30}$.

Em outro estudo sobre os fatores relacionados à prematuridade, demostrou-se maior ocorrência de prematuridade nas faixas etárias extremas e concluiu-se que a idade 
materna avançada pode agir independentemente, ou associada a agentes conflitantes como as doenças crônicas ou fatores sociais, culturais e demográficos ${ }^{13}$.

Ressalta-se que a escolaridade é comumente associada à prematuridade. Há maior risco, já evidenciado por pesquisas científicas, de nascimento prematuro nas mulheres com menor escolaridade ${ }^{13}$.

Quanto à gemelaridade, no presente estudo, apenas uma gestação múltipla foi observada. O risco de prematuridade encontra-se aumentado em situações de gestações múltiplas e, assistência pré-natal inadequada ${ }^{13}$. Sabe-se também que a assistência prénatal que resulta em melhores desfechos perinatais deve incluir: condutas acolhedoras; ações educativas e preventivas; detecção precoce de patologias e de situações de risco gestacional; estabelecimento de vínculo entre os serviços; e fácil acesso a serviços de saúde, que favorecem a sua qualidade e efetividade ${ }^{31}$.

Quanto às intercorrências obstétricas, $85,7 \%$ das mulheres deste estudo apresentaram pelo menos uma intercorrência na gestação, com proporção relevante da hipertensão (42,9\%), não restrita à doença hipertensiva específica da gravidez (DHEG). A incidência esperada de DHEG é entre 5 a $10 \%{ }^{19}$. Contudo, assim como no presente estudo, outros estudos evidenciaram percentis maiores de DHEG e intercorrências relacionadas a hipertensão na gestação, a saber $23,8 \%{ }^{19}$ e $17,0 \%{ }^{24}$.

Uma pesquisa observacional transversal, realizada em São Paulo, apontou a hipertensão arterial como um dos principais fatores de risco para parto prematuro. A hipertensão arterial é a complicação mais comum da gravidez e a principal causa de morbimortalidades materna e perinatal e, consequente, hospitalização em UTIN ${ }^{30}$.

Já em UTI obstétrica de um hospital terciário do Recife, Pernambuco, entre 2007 e 2010, foram identificados casos de near miss materno devido a distúrbios hipertensivos $(62,7 \%)$, elevando a razão de mortalidade materna e morbimortalidade perinatal20. Entende-se que o adequado e seguro enfrentamento das complicações ocorridas no ciclo grávido puerperal tem repercussão como estratégia de combate à mortalidade materna.

Quanto às medicações utilizadas, quase $80 \%$ das mães fizeram o uso de corticoide antenatal. A corticoterapia para as gestantes em risco de parto prematuro é fortemente recomendada, objetiva o amadurecimento pulmonar fetal, reduz a ocorrência de morbidades, a mortalidade neonatal, a necessidade de suporte ventilatório e as admissões em UTIN, melhorando a sobrevida do RN. Em cerca de 70 a $80 \%$ das gestações interrompidas até a $34^{\mathrm{a}}$ semana, as mulheres recebem a corticoterapia antenatal ${ }^{3,32}$.

Por fim, considera-se relevante a constância na recomendação científica pela qualificação da atenção hospitalar ao parto e nascimento, por se tratar de uma estratégia 
prioritária na redução de desfechos desfavoráveis, entre eles o parto prematuro, near miss materno e neonatal e óbitos evitáveis. E, ainda, com esta iniciativa se colabora com o cumprimento de alguns dos Objetivos de Desenvolvimento Sustentável (ODS) globais, especificamente os seguintes: Saúde e bem estar (no 3); Igualdade de gênero (no 5); Redução das desigualdades (no 10); Paz, justiça e instituições eficazes (no 16) ${ }^{33}$.

\section{CONCLUSÃO}

O planejamento cuidadoso, a coleta e a análise adequada dos dados aumentam a sua confiabilidade, conferindo validade interna aos mesmos. Os critérios de inclusão no presente estudo assemelham-se às populações de estudo e cenários similares, conferindo a possibilidade de validação comparativa externa.

Algumas limitações apresentadas neste estudo podem ser relacionadas como: as intrínsecas à utilização de dados secundários, coletados de prontuários, portanto não conferidas com as mães e profissionais a fidedignidade do registro; a exiguidade de outros estudos que permitissem a comparabilidade das informações geradas para uma discussão mais aprofundada dos resultados; e, a situação de calamidade na saúde, vivenciada atualmente com a COVID-19, que limitou o quantitativo esperado de RNPT para o estudo, mediante a redução da disponibilidade dos leitos intensivos neonatais. A coleta de dados cuidadosa e auxiliada por dupla checagem sempre que necessário, bem como, a inclusão de profissionais de saúde da área para a discussão dos mesmos, sempre que necessário, minimizaram as limitações citadas.

Reafirma-se que, devido ser multifatorial, o nascimento prematuro é uma preocupação mundial e contrasta com o crescente desenvolvimento tecnológico, que apresenta potencial de impacto para a redução da MI.

Neste estudo, as características neonatais e maternas verificadas são variadas, assim como o encontrado nos estudos desenvolvidos em outros cenários. Contudo, conhecê-las pode: favorecer maior (re)conhecimento do cenário do serviço hospitalar; apontamento das medidas para qualificá-los; o planejamento de ações para a prevenção dos agravos à saúde neonatal e materna, mais prevalentes no serviço, assim como, o near miss e os óbitos; a gestão dos leitos nas instituições hospitalares referência para alto risco, com provimento de tratamentos e cuidados eficazes e seguros; e, consequentemente, impacto na preservação de vidas. E ainda, este estudo é replicável em outros cenários semelhantes.

Por fim, acredita-se que as contribuições deste estudo poderão ser aplicados para a discussão de estratégias que reduzam o nascimento prematuro, a morbidade grave de gestantes e neonatos e, em especial, a mortalidade neonatal precoce. Recomendam-se estudos futuros com amostra e variáveis ampliadas, análises estatísticas, e métodos de 
pesquisa que gerem evidências científicas com elevado grau de recomendação, para o melhor embasamento científico dos serviços e da assistência materna e neonatal.

\section{REFERÊNCIAS}

1. World Health Organization (WHO). Como melhorar os desfechos clínicos nos partos prematuros [Internet]. 2015 [citado em 2021 abr 06]. Disponível em: http://apps.who.int/iris/bitstream/10665/204270/14/ WHO-RHR-15.22-por.pdf

2. World Health Organization (WHO). Preterm birth [Internet]. Geneva: WHO; 2018 [citado em 2019 set 02]. Disponível em: https://www.who.int/en/news-room/fact-sheets/detail/preterm-birth

3. Oliveira MG, Viau ÂC, Heidemann LA, Nicoloso L, Volkmer DFV. Mortalidade neonatal precoce em recém-nascidos de muito baixo peso: estudo de coorte. Bol científico Pediatr [Internet]. 2015 [citado em 2020 mai 02];04(3):75-9. Disponível em: http://dx.doi.org/2238-0450/15/04-03/75.

4. Ribeiro JF, Silva LLC, Santos IL, Luz VLES, Côelho DMM. O prematuro em unidade de terapia intensiva neonatal: a assistência do enfermeiro. Rev enferm UFPE on line [Internet]. 2016 [citado em 2019 set 02];10(10):3833-41. Disponível em: http://dx.doi.org/10.5205/reuol.9667-87805-1-ED1010201615.

5. Sociedade Brasileira de Pediatria (SBP). Novembro: Mês da Prevenção da Prematuridade [Internet]. 2019 [citado em 2021 abr 06]. Disponível em: https://www.sbp.com.br/fileadmin/user_upload/DocCientNeonatol-SBP_Prematuridade_18112019___.pdf

6. Ahumada-Barrios ME, Alvarado GF. Fatores de Risco para parto prematuro em um hospital. Rev LatinoAm Enfermagem [Internet]. 2016 [citado em 2021 abr 06]; 24:e2750. Disponível em: https://www.revistas. usp.br/rlae/article/view/124536/121035

7. Fialho FA, Dias IMÁV, Silva LR, Santos RS, Salvador M. Tecnologias aplicadas pela enfermagem no cuidado neonatal. Rev baiana enferm [Internet]. 2015 [citado em 2019 set 02];29(1):23-32. Disponível em: https://portalseer.ufba.br/index.php/enfermagem/article/view/12309/9538

8. Tamez RN. Enfermagem na UTI neonatal: assistência ao recém-nascido de alto risco. Rio de Janeiro: Guanabara Koogan; 2013.

9. Cuschieri S. The STROBE guidelines. Saudi J Anaesth [Internet]. 2019 [citado em 2020 abr 27]; 13(Suppl 1):S31-S34. Disponível em: http://dx.doi.org/10.4103/sja. SJA_543_18

10. Rede Brasileira de Pesquisas Neonatais. Universidade Federal do Paraná / Hospital de Clínicas (UFPR - HC) [Internet]. [citado em 2020 abr 27]. Disponível em: http://www.redeneonatal.fiocruz.br/index.php/ufpr-hc

11. Organização Mundial da Saúde (OMS). CID-10 Classificação Estatística Internacional de Doenças e Problemas Relacionados à Saúde. 10a rev. São Paulo: Universidade de São Paulo; 1997. vol.1 e vol.2.

12. BRASIL. Resolução $n^{\circ} 466$, de 12 de dezembro de 2012. Institui as diretrizes e normas regulamentadoras de pesquisas envolvendo seres humanos. Disponível em: https://bvsms.saude.gov.br/bvs/saudelegis/ cns/2013/res0466_12_12_2012.html

13. Oliveira LL, Gonçalves AC, Costa JSD, Bonilha ALL. Maternal and neonatal factors related to prematurity. Rev Esc Enferm USP [Internet]. 2016 [citado em 2020 abr 15]; 50(3):382-9. Disponível em: http://dx.doi. org/10.1590/S0080-623420160000400002

14. Dias JPV, Costa MC, Sette DS, Nobre LN. Perfil clínico de neonatos internados em uma Unidade de Terapia Intensiva Neonatal. Brazilian Journal of Development [Internet]. 2019 [citado em 2020 mai 15]; (5):22296-309. Disponível em: http://dx.doi.org/10.34117/bjdv5n10-356

15. World Health Organization (WHO). Declaração da OMS sobre Taxas de Cesáreas [Internet]. Geneva: WHO; [2015] [citado em 2020 abr 15]. Disponível em: https://apps.who.int/iris/bitstream/ handle/10665/161442/WHO_RHR_15.02_por.pdf?sequence=3

16. Downe S. Reducing routine interventions during labour and birth: first, do no harm. Cad. Saúde Pública [Internet]. 2014 [citado em 2020 mai 10]; 30(Suppl.):S17-S47. Disponível em: http://dx.doi. org/10.1590/0102-311XCO04S114 
17. Domingues RMSM, Dias MAB, Nakamura-Pereira M, Torres JA, d'Orsi E, Pereira APE, et al. Process of decision-making regarding the mode of birth in Brazil: from the initial preference of women to the final mode of birth. Cad. Saúde Pública [Internet]. 2014 [citado em 2020 mai 05]; 30(Suppl 1):S101-S116. Disponível em: http://dx.doi.org/10.1590/0102-311X00105113.

18. Silva AAM, Leite AJM, Lamy ZC, Moreira MEL, Gurgel RQ, Cunha AJLA, et al. Neonatal near miss in the Birth in Brazil survey. Cad. Saúde Pública [Internet]. 2014 [citado em 2020 mai 10]; 30(Suppl 1): S182-S191. Disponível em: http://dx.doi.org/10.1590/0102-311X00129613.

19. Buendgens BB, Teles JM, Gonçalves AC, Bonilha ALL. Características maternas na ocorrência da prematuridade tardia. Rev enferm UFPE online [Internet]. 2017 [citado em 2020 abr 15];11:2897-906. Disponível em: http://dx.doi.org/10.5205/reuol.11007-98133-3-SM.1107sup201711

20. Oliveira LC, Costa AAR. Fetal and neonatal deaths among cases of maternal near miss. Rev Assoc Med Bras [Internet]. 2013 [citado em 2020 mai 10];59(5):487-94. Disponível em: http://dx.doi.org/10.1016/j.ramb.2013.08.004

21. Chaves RL. Birth as a radical experience of change. Cad. Saúde Pública [Internet]. 2014 [citado em 2020 mai 10]; 30(Suppl. 1), S14-S16. Disponível em: http://dx.doi.org/10.1590/0102-311XPE03S114

22. Instituto Brasileiro de Geografia e Estatística (IBGE). Estatísticas do Registro Civil [Internet]. 2019 [citado em 2020 abr 15]. Disponível em: https://sidra.ibge.gov.br/tabela/2680

23. Lansky S, Fiche AAL, Silva AAM, Campos D, Bittencourt SDA, Carvalho ML, et al. Birth in Brazil survey: neonatal mortality, pregnancy and childbirth quality of care. Cad Saude Publica [Internet]. 2014 [citado em 2020 mai 10]; 30(Suppl. 1): S192-S207. Disponível em: http://dx.doi.org/10.1590/0102-311X00133213

24.SociedadeBrasileiradePediatria(SBP). ReanimaçãodoPrematuro<34semanasemsaladeparto:Diretrizes 2016 da Sociedade Brasileira de Pediatria [Internet]. 2016 [citado em 2020 abr 15]. Disponível em: https://www. sbp.com.br/fileadmin/user_upload/DiretrizesSBPReanimacaoPrematuroMenor34semanas26jan2016.pdf

25. Aquino RAN. Efeito da nutrição parenteral precoce em recém-nascidos pré-termos de muito baixo peso o nascer [dissertação]. Uberlândia: Universidade Federal de Uberlândia; 2014.

26. Zanella A. Influência da nutrição enteral na microbiota intestinal do recém-nascido pré-termo [dissertação]. Rio Grande do Sul: Universidade Federal do Rio Grande do Sul; 2019.

27. Valentine CJ, Fernandez S, Rogers LK, Gulati P, Hayes J, Lore P, et al. Early amino-acid administration improves preterm infant weight. Journal of Perinatology [Internet]. 2009 [citado em 2020 mai 17]; 29(6):42832. Disponível em: http://dx.doi.org/10.1038/jp.2009.51

28. Rochow N, Fusch G, Mühlinghaus A, Straube S, Utzig N, Fusch C, et al. A nutritional program to improve outcome of very low birth weight infants. Clinical Nutrition [Internet]. 2012 [citado em 2020 mai 17]; 31(1):124-31. Disponível em: http://dx.doi.org/10.1016/j.clnu.2011.07.004

29. Instituto Brasileiro de Geografia e Estatística (IBGE). População residente, por cor ou raça, segundo a situação do domicílio, o sexo e a idade [Internet]. 2010 [citado em 2020 abr 15]. Disponível em: https:// sidra.ibge.gov.br/Tabela/3175

30. Berger AZ, Zorzim VI, Pôrto EF, Alfieri FM. Premature childbirth: pregnant women's characteristics of a population in the South area of São Paulo. Rev. Bras. Saúde Matern. Infant. [Internet]. 2016 [citado em 2020 mai 17]; 16(4):437-45. Disponível em: http://dx.doi.org/10.1590/1806-93042016000400005

31. Viellas EF, Augusto M, Dias B, Viana J, Bastos MH. Prenatal care in Brazil. Cad Saude Publica [Internet]. 2014 [citado em 2020 mai 05]; 30(Suppl. 1), S85-S100. Disponível em: http://dx.doi.org/10.1590/0102-311X00126013

32. Carelli MCB. Efeitos da corticoterapia antenatal sobre a hemodinâmica do feto sob risco de parto prematuro [dissertação]. Rio de Janeiro: Instituto Nacional de Saúde da Mulher, da Criança e do Adolescente Fernandes Figueira; 2018.

33. Nações Unidas Brasil (ONU Brasil). Transformando Nosso Mundo: A Agenda de 2030 para o Desenvolvimento Sustentável [Internet]. 2015 [citado em 2021 abr 08]. Disponível em: https://nacoesunidas. org/pos2015/agenda2030/ 